\title{
Identification of PVCs based on Peak detection with Teager Energy Operator
}

\author{
V. Sharmila \\ Department of ECE, \\ Kamala Institute of Technology \& Science, \\ Karimnagar, India,
}

\author{
K.Ashoka reddy \\ Dept.of E \&l Engineering, \\ Kakatiya Institute of Technology \& Science, \\ Warangal, India
}

\begin{abstract}
Teager Energy Operator (TEO) is a nonlinear energy operator that can be used to analyze the biomedical signals like ECG from energy point of view. Presence of Premature Ventricular Contraction (PVC) beats in an ECG record can be easily identified from normal beats using the proposed TEO model. TEO tracks the instantaneous envelope of $\mathrm{R}$ peak, which clearly illustrates whether the trigger pulse for depolarization has originated from the SA node (for normal beat) or from the ventricles (for PVC beat). Based on the above analysis if the tracked envelope corresponds to an impulsive spike, it can be identified as the $\mathrm{R}$ peak of a normal beat and if it is distorted it can be identified as the one corresponding to the $\mathrm{R}$ peak of PVC beat. Reliability of the proposed method is high compared to other PVC identification methods based on peak detection. The algorithm applied to a set of PVC data taken from MIT-BIH database had resulted in measures of, greater than $99 \%$ of sensitivity, positive predictivity of $100 \%$ and detection error rate less than $.03 \%$.
\end{abstract}

Keywords: ECG, PVC, TEO, sensitivity, positive predictivity and detection error.

\section{INTRODUCTION}

Evolution of computer technology led to the development of many software algorithms for QRS complex detection. These software algorithms have reduced the hardware complexity required earlier for the QRS detection. ECG waveform consists of three significant features such as $\mathrm{P}$ wave, QRS complex and T-wave. $\mathrm{P}$ wave is a low frequency, low amplitude feature obtained due to the atrial depolarization which is the first positive deflection on the ECG waveform. QRS complex consists of first negative deflection treated as $\mathrm{Q}$-wave preceding $\mathrm{R}$ wave, due to the depolarization of inter ventricular septum, and $\mathrm{R}$-wave is again maximum positive deflection due to ventricular depolarization in the ECG waveform, and $\mathrm{S}$ wave is a negative deflection due to late depolarization of some ventricular areas. QRS complex is the prominent feature required for heart diagnosis. Rest of the paper is organized as follows : QRS detection and its significance is discussed in 1.1, PVC beat is discussed in 1.2, nonlinear Teager Energy(TE) operator is described in section 2 and the proposed TEO method for PVC identification based on peak detection is discussed in section 3. Results and discussion in section. 4 and conclusions in section.5.

\subsection{QRS detection}

QRS detection required for cardiac diagnosis is entirely computer based. Strong energy is required by Sino Atrial (SA) node for ventricular depolarization to occur, which results in normal ECG beat termed as normal sinus rhythm data, whereas when SA node fails to trigger the pulses, its functioning is taken up by the other parts of myocardium such as ventricles or atria called as ectopic beat. PVC beat occurs when the trigger pulse required for depolarization is initiated by the ventricles. For a PVC beat, QRS complex is wide, bizarre with no preceding P-wave. ECG waveform being a low frequency signal $(.05-100) \mathrm{Hz}$ gets easily contaminated with the PLI noise, baseline wander and other artifacts while recording which makes accurate peak detection a difficult task. Many algorithms were presented in the literature for peak detection and arrhythmia classification. Gary Friesen et al [1] have implemented nine different algorithms based on first, second order derivatives and digital filters for QRS peak detection and observed that none of them were able to detect all QRS peaks without false positives. J.Pan and W.J.Tompkins [2] have developed a real time QRS detection algorithm employing adaptive thresholding for reliable QRS detection and achieved a sensitivity of $99.3 \%$. This algorithm proved to be effective in accurate peak detection under noisy conditions also at the cost of computational complexity. Ivo Iliev et al [3] devoted his work for development of a QRS detector with less memory requirement to identify the actual QRS peak position from the deviated one. Paul S.Addison [4] has studied the use of continuous wavelet transform and discrete wavelet transform for the analysis and extraction of features like QRS complex from nonstationary signals like ECG, and suggested it as a powerful tool for diagnosing cardiac pathology. O.Dwyer et al [5] proved that arrhythmia classification is possible based on QRS width.

\subsection{Premature Ventricular Contraction}

Premature ventricular contraction occurs when the heartbeat is initiated by the purkinje fibres present in the ventricles rather than the sino-atrial node. Decreased oxygen supply is the reason for PVCs to occur where the ventricles contract before the atria fills the ventricles with blood and is, less dangerous if a single PVC beat appears in the ECG. Risk of sudden cardiac death prevails if PVC beats with different configurations of QRS complexes occur continuously in the ECG. $F$ (fusion of $P V C$ with normal beat), $V(P V C$ beat) and $N($ normal beat $)$ in the from the ECG record-208 of ML-II taken from MIT-BIH database is shown in the fig. 1 . 


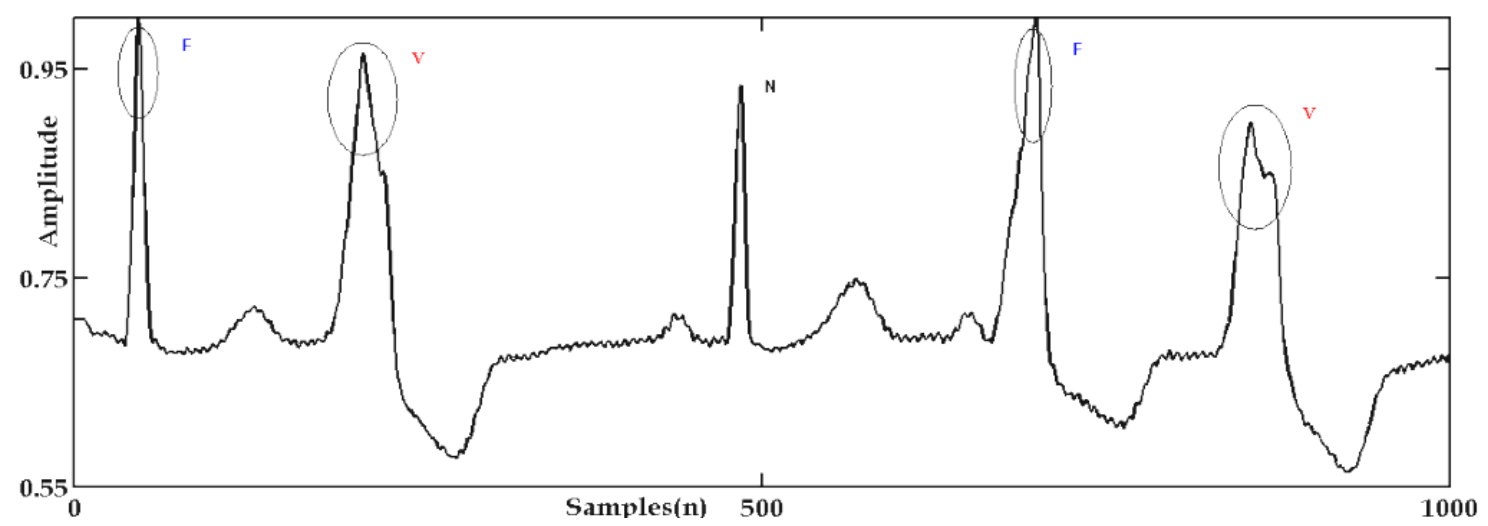

Figure 1.Record 208 with normal, fusion and PVC beats.

Occurrence of PVC's in the ECG signal alters the Morphological features, due to which peak detection is further complicated. Many algorithms were developed in the literature for PVC identification. Murthy et al [6] proved that, PVC data can be identified from normal data based on minimum phase correspondent (MPC). J.S.Paul et al [7], by using the concepts of DCT in conjunction with homomorphic or cepstral filtering have identified PVCs under noisy conditions upto 10dB. Bert.Uwe, et al [8] have developed different algorithms using derivatives, digital filters, wavelets and Hilbert transform techniques for QRS detection and discussed the comparative results. D.Benitez et.al [9] has used the Hilbert transform properties to find the envelope of ECG beat which reflects the QRS peak. The proposed work uses a nonlinear Teager Energy Operator (TEO) in comparison with the Hilbert transform for PVC identification based on peak detection.

\section{TEAGER ENERGY OPERATOR A NON-LINEAR ENERGY FUNCTION}

Conventional Fourier transform acts as a linear energy operator for the energy extraction from a linear signal, which is phase blind. Many linear operators are presented in the literature earlier for signal analysis, which mainly concentrated on linear components of the signal which made them unsuitable for the analysis of nonlinear signals like speech and ECG signals. This motivated Eivind Kvedalen [10] to introduce a nonlinear energy operator TEO, which can be applied to nonlinear signal analysis. Petros and Potamianos [11] have found that TEO, a Teager-Kaiser (1990) operator is a higher order discrete energy operator that can be applied to nonlinear signals, which extracts the energy required by the source to generate the signal. Important property of TEO is it reflects the energy of the system rather than the energy of the signal as per Kaiser J.F [12]. C.Kamath [13] from his study about TEO, found that energy extracted from nonlinear signals, leads to successful classification of arrhythmia beats from normal beats. Main advantage of Teager energy operator is that it is a nonlinear energy operator used for extracting the energy of the source that generated the signal based on physical and mechanical conditions of the system instead of extracting the energy of the signal. Energy is distributed over the entire frequency band of the signal. Mathematically energy of a signal is calculated by squaring the absolute value of the Fourier transform of a signal.

$$
E=|X(k)|^{2}
$$

According to Kaiser energy required to generate a nonlinear signal varies as a function of both amplitude as well as frequency whereas energy required to generate a linear signal varies as a function of amplitude only. Teager Energy (TE) concept was derived from the solution of a second order differential equation which describes the functioning of a nonlinear signal.

Sino-atrial node present at the top right corner of the heart acts as a natural pacemaker which continuously fires the impulses. These electric impulses allow both the atria and ventricles to undergo depolarization and repolarization activities. According to Kaiser this action can be represented by a second order differential equation given below as

$$
\frac{d^{2} x}{d t^{2}}+\frac{k}{m} x=0
$$

Where $x(t)$ is the generated ECG signal with respect to time, $m$ is the mass of the heart and $\mathrm{k}$ is a constant. Rhythmic heart beat due to the depolarization and repolarisation activities is the solution of equation (2) given as

$$
x(t)=A \cos (\omega t+\varphi)
$$

where $\mathrm{A}$ is the amplitude with initial phase $\varphi$. Similarly $x(n)$ is the generated ECG signal in discrete domain represented as

$$
x(n)=A \cos (\Omega n+\Phi),
$$

$\Omega$ is the digital frequency in radians/sample given as $\Omega=2 \pi f / f s, f$ is the analog frequency and $\Phi$ is the initial phase. $\Omega$, f, $\Phi$ are the three unknown parameters and $f s$ is the sampling frequency in $\mathrm{Hz}$. The solution to find the three unknown's is given by using only three samples $x(n), x(n-1)$ and $x(n+1)$. Now the energy at any given instant of time $\mathrm{n}$ is given as

$$
\begin{aligned}
& E[n]=x(n)^{2}-x(n-1) x(n+1) \\
& =A^{2} \sin ^{2}(\Omega) \\
& E[n] \square A^{2} \Omega^{2} \text { for } \Omega \text { small }
\end{aligned}
$$

The above energy equation works well under the condition of positive $\Omega$ less than one quarter of the sampling frequency which accounts for an approximation error of less than $11 \%$. 
The above concept defines TEO as the squared product of instantaneous amplitude and corresponding frequency. TEO measurement is instantaneous as it uses just three samples for energy extraction and is suitable for real time applications. TEO has an important characteristic of time resolution with which it tracks the continuous changes occurring in the energy (squared product of amplitude and frequency) of the signal considering the phase information also. Using the concepts of TEO, QRS peak is extracted from the envelope obtained by teager energy operator. Teager energy reflects the changes occurring in the envelope of the ECG signal. Hari Mohan Rai et.al [14] in their paper has proved the efficiency of wavelet transforms in denoising the non-linear ECG signals with different mother wavelets. Rajni et.al [15] has given an overview of various methods like wavelet transforms FFT techniques for the ECG signal analysis.
Hilbert transforming a time domain signal does not bring about a change in domain i.e, transformed signal remains in same time domain. When a signal is Hilbert transformed all of its frequency components undergo a phase shift of 90 degrees where as amplitude remains unaltered. Hilbert transformer can be used as QRS peak detector

\subsection{New approach using TEO model:}

Block diagram of the proposed algorithm using TEO model for, PVC identification based on $\mathrm{R}$ peaks is shown in the fig.2.below. Sequence of steps involved in the algorithm is discussed below. Preprocessing is the first stage of the algorithm where average value of the ECG signal is subtracted from the samples of the signal to eliminate the effect of DC noise.

\section{METHODS}

\subsection{Hilbert Transform}

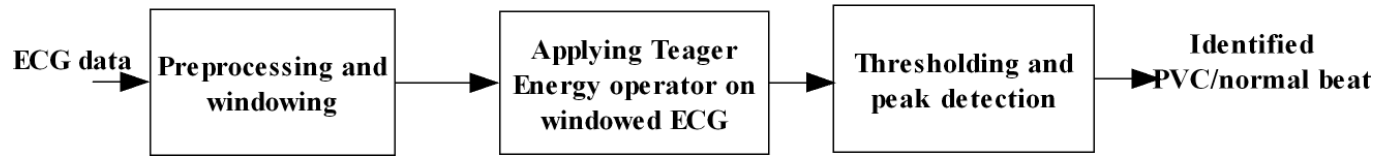

Figure 2. Block diagram of the proposed scheme.

Nonlinear TEO model works effectively with short segments of data, so the required ECG data is divided into short segments using windowing technique, which also eliminates the edge artifacts. Hanning window of size 1000 samples is used to segment the preprocessed data. TEO extracts the energy of SA node required to trigger the impulses for ventricular depolarisation which generates action potential. This action potential represents the envelope of ECG beat.

\section{RESULTS AND DISCUSSION}

Teager energy operator is used to extract the energy from the source (SA node) of the signal which generated the action potential. TEO follows the instantaneous changes occurring in the envelope of ECG signal that represents the QRS peaks even in the presence of PLI and baseline wander noises. Normal beat is generated by the excitation of energy from SA node, whereas a PVC beat is generated by Purkinje fibers, which is low amplitude envelope as depicted by the TEO. The ability of TEO in peak detection is also verified with the real time ECG data acquired from various subjects in the
Position of the envelopes corresponds to the $\mathrm{R}$ peaks of normal beats and PVC beats. Thresholding these detected $\mathrm{R}$ peaks results in the proper identification of PVC beats from normal beats. Threshold value is set as given below.

$$
\text { Threshold }=\frac{\text { Maxvalue }- \text { Minvalue }}{2}
$$

laboratory as shown in fig.3. For this purpose MP150C data acquisition system of BIOPAC make is used along with the ECG100C amplifier for recording of a three lead ECG (Lead I, II and III), with a sampling frequency of $125 \mathrm{~Hz}$. Ten healthy volunteers were identified and with their informed prior consent, a three lead ECG data was recorded in the supine position. The recorded data is then used for the experimentation in the MATLAB 2007R environment. Energy extracted by TEO is simply following the QRS complex which illustrates that the recorded ECG is normal as shown in fig.4.
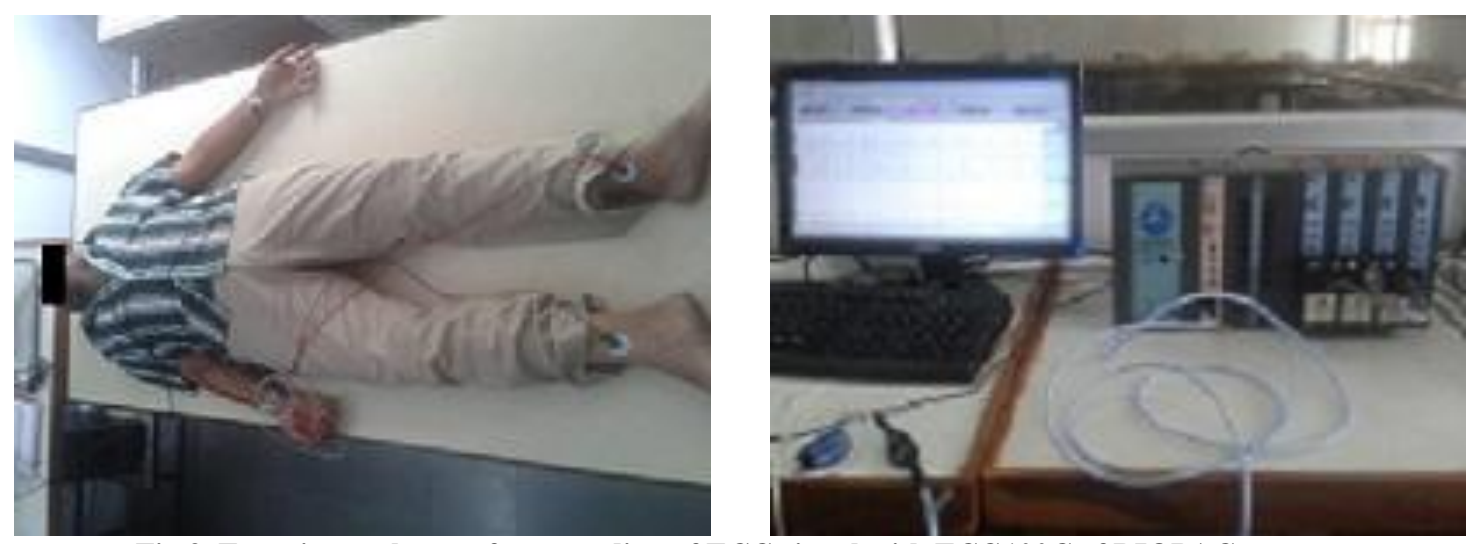

Fig 3. Experimental setup for recording of ECG signal with ECG100C of BIOPAC system. 


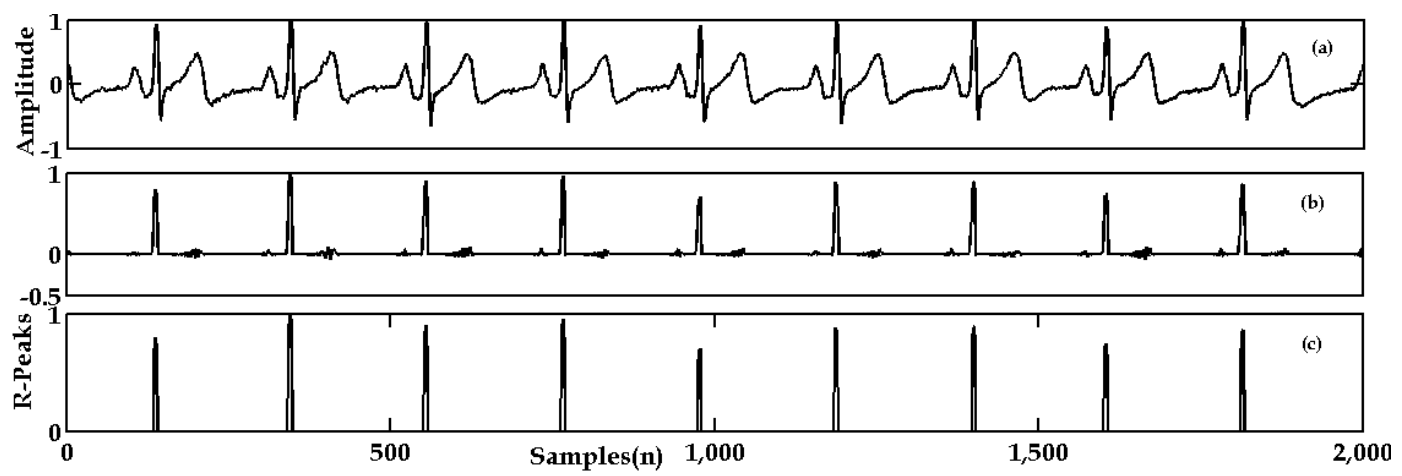

Figure 4. Online recorded ECG with normal beats in trace (a); $R$ peaks with the proposed method in trace (b) and thresholded $R$-peaks in trace(c).

Envelopes extracted by TEO for PVC beat is having low amplitude and exhibits heavy ripples (negative peaks) which implies that the beat is premature, and the envelope extracted for a normal beat takes the shape of an impulsive spike as shown in figs.5-6 for records 119 and 208 taken from MITBIH arrhythmia data base. Thresholding the envelopes obtained by TEO, PVC beats can be easily identified from normal beats based on detected peaks. Peaks corresponding to normal beats appear as impulsive spikes and peaks corresponding to the PVC beats disappear due to their low amplitude level less than the threshold value. Teager energy that represents the envelope of QRS peak gets extended in time domain for a PVC beat than a normal beat. This algorithm provides an easier way for PVC identification based on peak detection with high performance measures, when compared to the Hilbert transform method. It works effectively in identifying PVCs even in the presence of noise with less computational time than the other PVC identification methods based on peak detection.

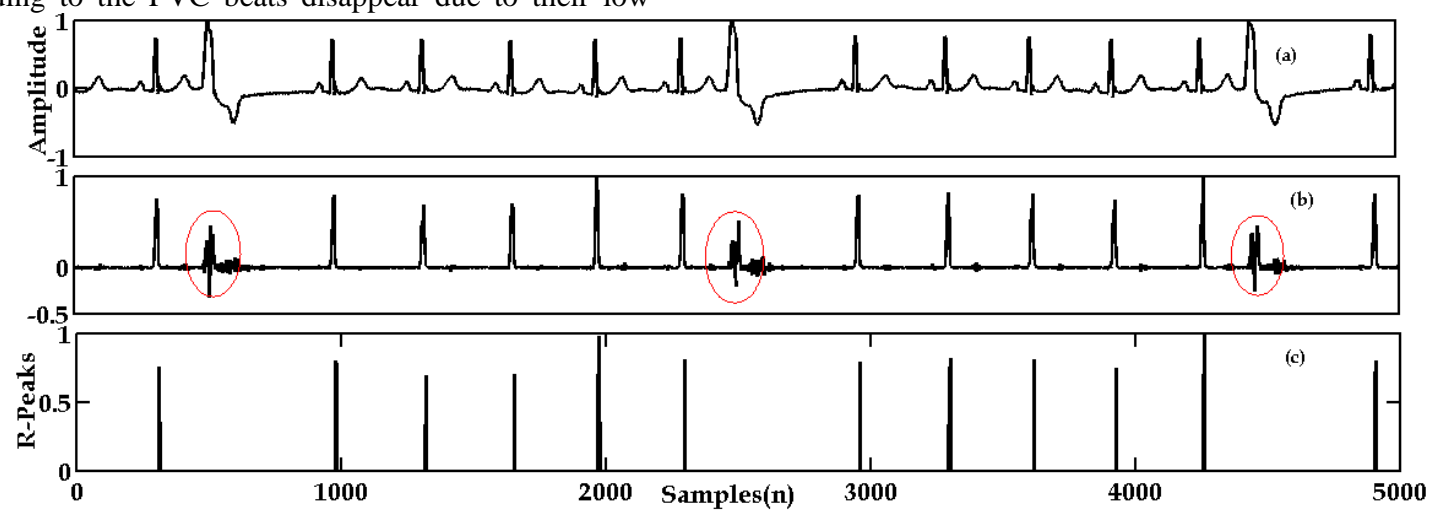

Figure 5.119 record with PVC beats encircled in trace (a); R-Peaks for PVC beats encircled in trace (b) and thresholded R-peaks only for normal beats in trace(c).

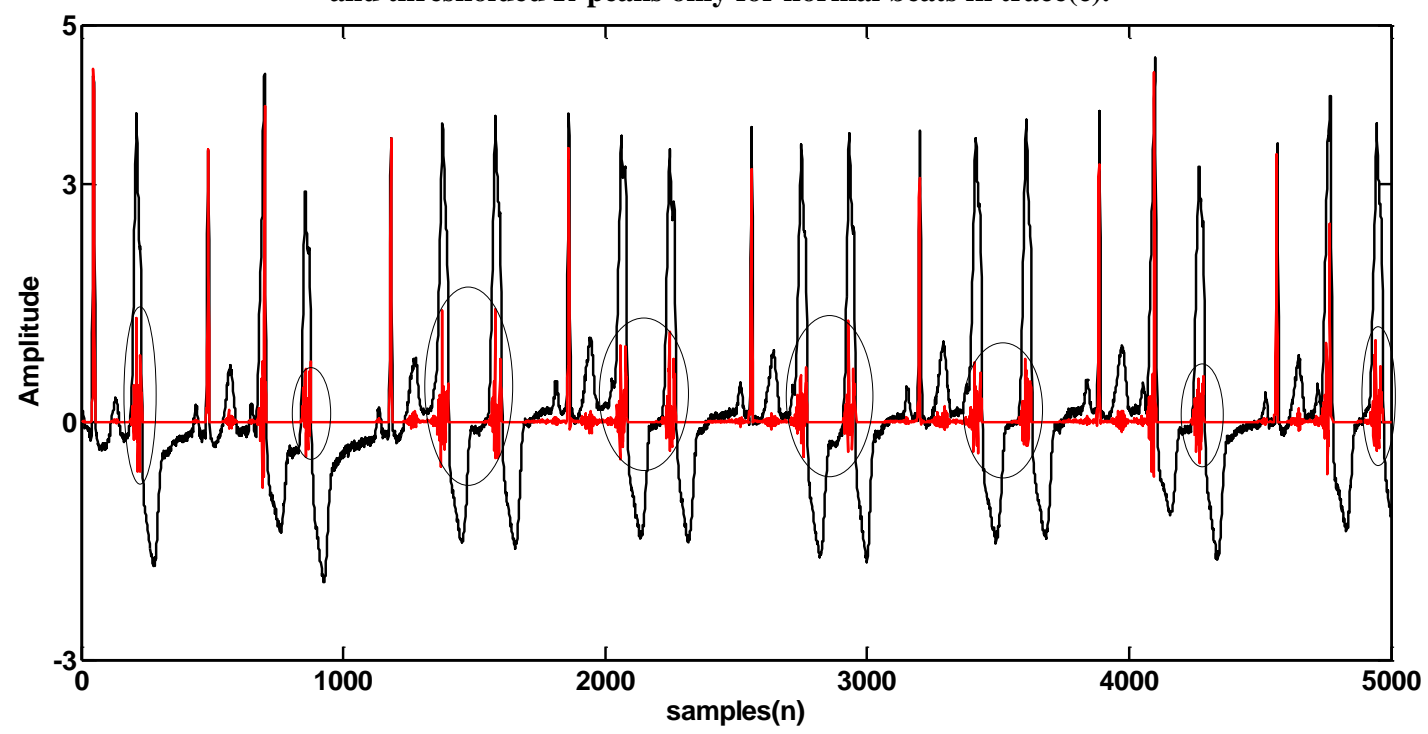

Fig 6.208 record with normal beats and PVC beats with corresponding R peaks and PVC beats with distorted peaks are encircled 

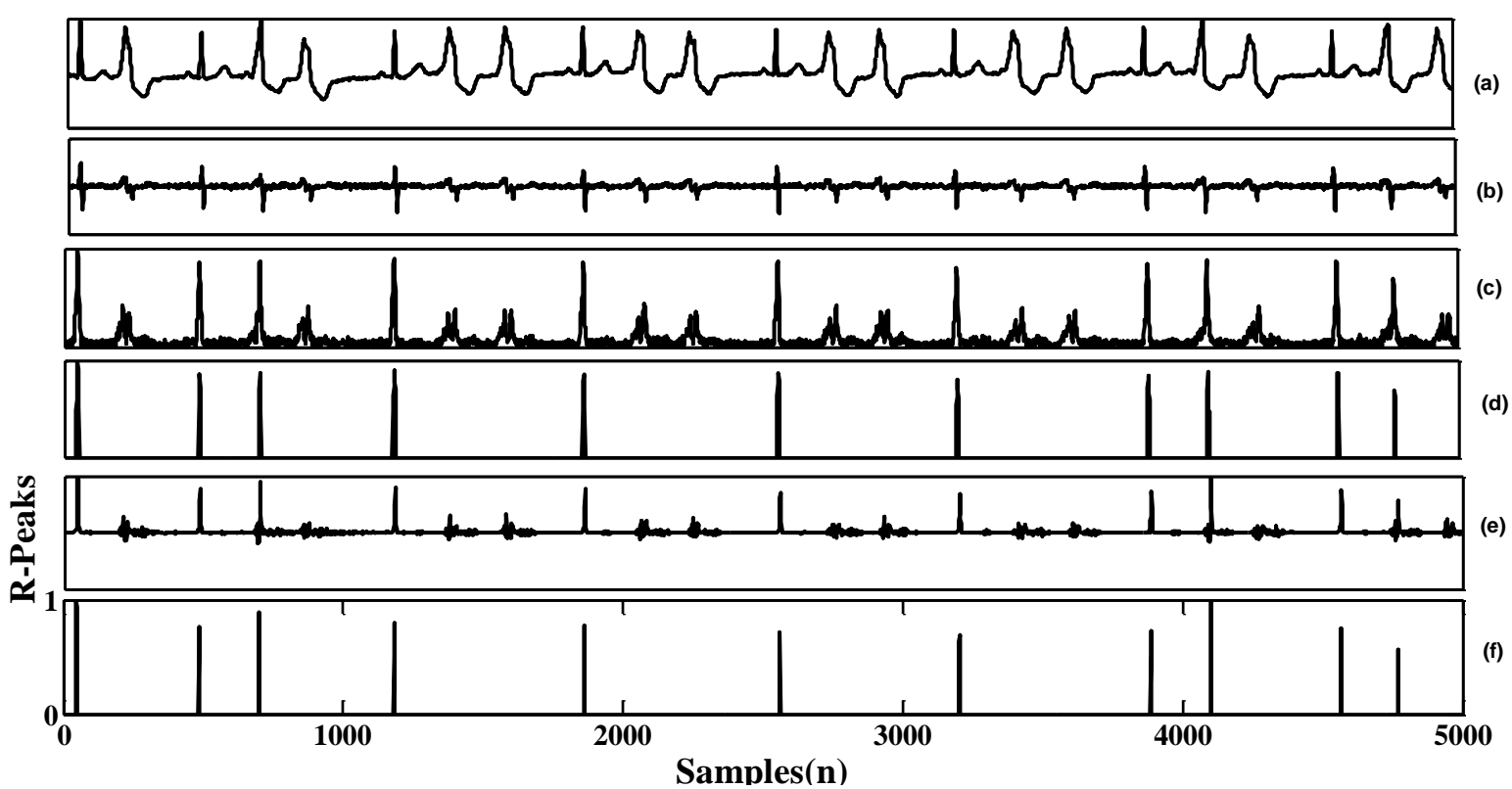

Figure 7. PVC record in trace(a); d/dt(ECG) is the first differential of ECG in trace(b)Hilbert transform of (b) in trace(c); thresholded R-Peaks from (c) in trace(d); teager energy in trace(e) and thresholded $R$ peaks in trace(f).

PVC identification using both the Hilbert transform method and TEO method are shown in fig.7. R-peak detection using Hilbert transform method requires, first differentiation of the ECG signal, then hilbert transform of the differentiated ECG signal extracts the envelope. Thresholding the envelopes results in impulsive spikes corresponding to normal beats positioning the R-peaks and, no peaks corresponding to low amplitude envelope of the PVC beats. Based on the above analysis PVC beats can be identified from normal beats. Comparitively TEO simply follows the envelope of ECG beats and thresholding the envelopes results in impulsive spikes for normal beats, fusion beats and no peaks for PVC beats which reveals that accurate PVC identification based on peak detection, with less computational time is possible with TEO method.

The proposed TEO model is applied to a set of PVC records and normal records taken from MIT-BIH arrhythmia database. Performance measures like Sensitivity (Se), Positive prediction $(+\mathrm{P})$, detection error rate were calculated with the given equations:

Sensitivity (Se): Sensitivity is the ability of the algorithm in detecting the actual peaks out of total peaks as given in the annotation file of MIT-BIH record. A false negative(FN) occurs when the actual QRS peak or a true beat, is not detected by the algorithm and a false beat detection is considered as false Positive (FP) and true positive (TP) is number of $\mathrm{R}$ peaks detected correctly.

$$
\text { Sensitivity }(\%)=\frac{T P}{T P+F N} \%
$$

Positive prediction $(+P)$ : It is the ability of the algorithm in predicting the true $\mathrm{R}$ peaks of normal beats from the total peaks given in the annotation file.

$$
\text { Positive Predictivity }(\%)=\frac{T P}{T P+F P} \%
$$

Detection error rate: low value of detection error indicates the efficacy of the algorithm in detecting the actual $\mathrm{R}$ peaks.

$$
\text { Detection errorr rate }(\%)=\frac{F P+F N}{\text { Total number of QRScomplex }} \%
$$

Nonlinear teager energy operator shows an excellent performance identification of PVC beats from normal beats with sensitivity of $99 \%,+\mathrm{P}$ of $100 \%$ and detection rate of $0.03 \%$ for 208 record. Teager energy in time domain represents the envelope of QRS peak. For a normal beat the teager energy exhibits an impulsive nature in the position of QRS peak, whereas for a PVC beat the QRS envelope consists of negative peaks which indicate the disturbances in the conduction path of impulses. The performance results for both normal and PVC beats are given in Table 1 and comparative results are shown in Table 2. Peaks detected for short segments of ECG data for a total of 20,000 samples is shown in fig. 8 . 


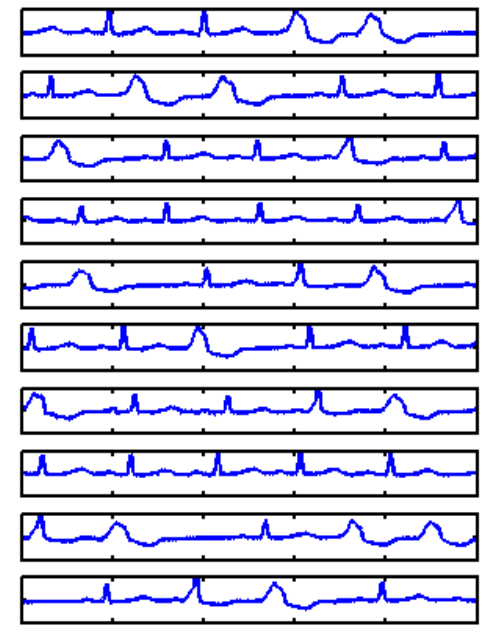

(a)

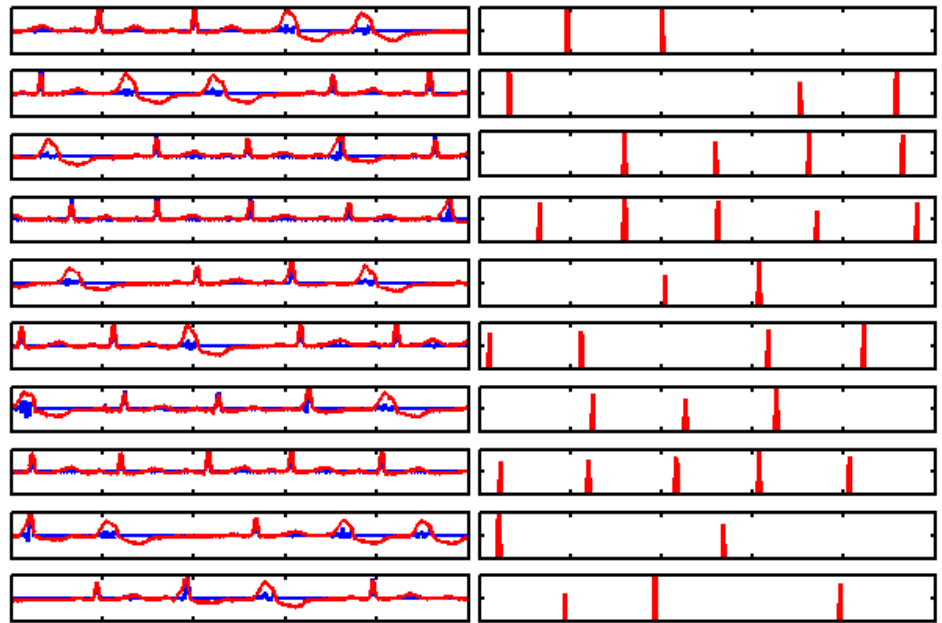

(b)

(c)

Fig.8. Segmented samples of record 208 in trace(a );overlapped ECG beats with extracted envelopes in trace(b); and thresholded R-peaks in trace(c) where R peaks for PVC beats are absent.

Table 1: QRS detection of PVC data from MIT-BIH database, with proposed algorithm

\begin{tabular}{|c|c|c|c|c|c|c|c|}
\hline $\begin{array}{l}\text { PVC } \\
\text { record }\end{array}$ & $\begin{array}{c}\text { Actual } \\
\text { number } \\
\text { of PVCs }\end{array}$ & $\begin{array}{l}\mathbf{F} \\
\mathbf{P}\end{array}$ & $\mathbf{F}$ & $\begin{array}{r}\text { Failed } \\
\text { detection }\end{array}$ & $\begin{array}{l}\mathrm{Se} \\
(\%)\end{array}$ & $\begin{array}{c}\text { Petection } \\
\text { error } \\
\text { rate } \\
(\%)\end{array}$ & $+\mathrm{P} \%$ \\
\hline 119 & 444 & 0 & 1 & 1.0 & 98 & \begin{tabular}{|l|}
0.03 \\
\end{tabular} & 100 \\
\hline 124 & 47 & 0 & 0 & 0 & 100 & 0.0 & 100 \\
\hline 200 & 826 & 0 & 1 & 1.0 & 99 & .03 & 100 \\
\hline 208 & 992 & 0 & 1 & 1.0 & 99 & .016 & 100 \\
\hline 221 & 396 & 0 & 0 & 0.0 & 100 & 0.0 & 100 \\
\hline 210 & 194 & 0 & 0 & 0.0 & 100 & 0.0 & 100 \\
\hline
\end{tabular}

Table 2: Performance comparison of the proposed algorithm with the Hilbert transform method

\begin{tabular}{|c|c|c|c|}
\hline Method & $\mathbf{S e}(\%)$ & $\begin{array}{c}\text { Detection } \\
\text { error rate } \\
(\%)\end{array}$ & $\mathbf{+ P \%}$ \\
\hline $\begin{array}{c}\text { Proposed } \\
\text { TEO }\end{array}$ & 99.8 & 0.016 & 100 \\
\hline $\begin{array}{c}\text { Hi1bert } \\
\text { transfor } \\
\text { m }\end{array}$ & 99.8 & 0.39 & 99.73 \\
\hline
\end{tabular}

\section{CONCLUSION}

This paper presents a nonlinear modeling algorithm using the characteristics of TEO for PVC Identification based on $\mathrm{R}$ peak detection. Using teager energy operator envelopes are extracted for the ECG beats form the records taken from MIT$\mathrm{BIH}$ database constituting the, normal beats, fusion beats and PVC beats. Thresholding the extracted envelopes results in an impulse spike for normal and fusion beats, which illustrates that the energy required for ventricular depolarization was originated from SA node. For a PVC beat, no peak was detected after thresholding as the extracted envelope is having low amplitude and ripples which indicate that the energy required for ventricular depolarization was generated by ectopic foci due to the failure of SA node (natural pacemaker). This algorithm is very simple using just only three samples compared to Hilbert transform based algorithm as given in Table-2. Results reveal that error detection rate using TEO model is less than that of Hilbert transform method. TEO model is proved to be computationally efficient in identifying the PVC beats from normal beats even in the presence of noise. This algorithm can be extended further for ECG enhancement and other arrhythmia identification.

\section{REFERENCES}

[1] Gary Friesen, M.Thomas Jannett,C.Manal Afify,J.Stanford Yates,:"A comparison of the noise sensitivity of nine QRs detection algorithms", IEEE trans.Biomed. Engg, 1990, 37, (1) ,pp.85-98

[2] J.Pan, W.J.Tompkins, "A real Time QRS detection algorithm”, IEEE Trans.Bio Med Eng. 44(1997), 159167. 10.1109/TBME.1985.325532.

[3] Ivo Iliev, Vessela Krasteva and Serafim Tabakov, "Realtime detection of pathological cardiac events in the electrocardiogram" 2007 Physiol. Meas. 28 259. DOI: $10.1088 / 0967-3334 / 28 / 3 / 003$

[4] Paul S Addison "Wavelet transforms and the ECG: a review" 2005 Physiol. Meas. 26 R155. DOI:10.1088/0967-334/26/5/R01

[5] O'Dwyer M.de.Chazal,P.Reily,R.B: "Beat classification for use in arrhythmia analysis", IEEE comp. cardiology., 2000, 27, Pp.395-398. DOI:10.1109/cic.2000.898540.

[6] Murthy ISN, Rangaraj MR, "New concepts for PVC detection". IEEE Trans on Bio Med Eng,1979;BME-26,409-415.

DOI: 10.1109/TBME.1979.326420

[7] J.S.Paul, M.R.S.Reddy and V.Jagadeesh Kumar "Identification of Premature Ventricular Cycles in Surface Electrograms Using the envelope of DCT" Applied Sig .Process (1998), Springer-Verlog, London, Pp.226-239.

[8] Bert.Uwe, K.,Carsten,H., Reinhold,O: "The principles of software QRS detection", IEEE Eng.Med.,Biol., 2002,21,(1),pp.42-57. DOI: 10.1109/51.993193

[9] D.Benitez, P.A. Gaydecki, A.Zaidi, A.P.Fitzpatrick "The use of Hilbert Transform in ECG signal analysis", Computers in Biology and Medicine 31(2001) 399-406. DOI: $10.1016 / \mathrm{S} 0010-4825(01) 00009-9$

[10] Eivind Kvedalen" Signal Processing Using the Teager Energy Operator and other nonlinear operators" ,May2003. 